\title{
Modelagem de uma Metodologia para Implementação de Aprendizagem Significativa em Ambientes Virtuais de Aprendizagem (AVAs)
}

\author{
Maria Eduarda Rebelo Di Carlli ${ }^{1}$, Tássia Laís Barros Bastos ${ }^{1}$, Bianca Carneiro \\ Ribeiro $^{2}$, Obionor de Oliveira Nóbrega ${ }^{1}$ \\ ${ }^{1}$ Departamento de Computação - Universidade Federal Rural de Pernambuco (UFRPE) \\ Recife - PE - Brasil \\ ${ }^{2}$ Unidade Acadêmica de Educação a Distância e Tecnologia - Universidade Federal \\ Rural de Pernambuco (UFRPE) \\ Recife - PE - Brasil \\ dicarllim@gmail.com, bastostlb@gmail.com, bianca.ribeiro@ufrpe.br, \\ obionor.nobregaeufrpe.br
}

\begin{abstract}
The increase use of ICT in the Distance Education modality requires the Virtual Learning Environments use methodologies and theories that enable a significant and active learning for students. This paper aims to describe a methodology for implementing significant learning theory in VLEs using structural planning of content and fixation activities. Resulting in a simplified implementation methodology for teachers to begin their virtual classrooms based on significant learning theory.
\end{abstract}

Resumo. Com o uso cada vez mais cotidiano das TDICs na modalidade de Educação a Distância, faz-se necessário que Ambientes Virtuais de Aprendizagem utilizem metodologias e teorias que possibilitem um aprendizado mais significativo e ativo para os alunos. Este artigo tem o objetivo de apresentar uma metodologia para implementação da teoria de aprendizagem significativas em AVAs independentemente da área de atuação do professor, por meio de um planejamento estrutural de conteúdos e atividades de fixação. Esta proposta possibilita uma implementação simplificada para professores iniciarem suas salas de aula virtuais baseadas na teoria de aprendizagem significativa.

\section{Introdução}

Ao considerar os avanços tecnológicos e as inevitáveis mudanças no processo educativo, é notável que a transformação digital tem sido expandida na área educacional com a finalidade de aprimorar o processo de ensino aprendizagem. A partir de recursos cada vez mais sofisticados e capazes de proporcionar experiências únicas, o ensino avança com o uso de estratégias digitais e, consequentemente, fortifica os caminhos para a Educação a Distância (EAD). Esta modalidade de ensino procura estimular e indagar os interesses dos seus estudantes frente à formação crítica e adequada a sua realidade, $\mathrm{o}$ 
que torna necessária uma educação problematizada que permita ao aluno a experimentação [Carvalho et al. 2018].

Neste cenário, a aprendizagem significativa é uma teoria que possibilita que a compreensão não linear de novos conhecimentos sejam adquiridos relacionando-se com o conhecimento prévio que o aluno possui, o que resulta em um desenvolvimento ativo de autonomia de pensamento e ação [Rolim e Scaramuzza 2016], sendo a esta creditada como elemento fundamental para a dinâmica entre as Metodologias Ativas e as Tecnologias em Mídias Digitais utilizadas em EAD [Silva et al. 2019].

Nestas condições, para que um processo de ensino seja eficiente, o design dos Ambientes Virtuais de Aprendizagem (AVA) e seus recursos são essenciais, mesmo se o conteúdo apresentado seja didaticamente repleto de significações, estas irão se perder se a linguagem e a organização dos materiais dispostos no ambiente virtual não forem adequadas ao público-alvo [Scaramuzza 2015]. Assim, diversas soluções com o uso de aprendizagem significativa e AVAs são propostas na literatura, porém, baseadas no uso de ferramentas do próprio ambiente ou soluções específicas elaboradas, não considerando a problemática da compreensão metodológica da sua implementação. Desta forma, existe a real necessidade de uma sistematização de como implementar a teoria de aprendizagem significativa em AVAs que resulte em planejamento estrutural de conteúdos e atividades de fixação significativos.

Ancorado nas lacunas proporcionadas no uso das ferramentas do AVA e no processo de ensino aprendizagem significativo, este artigo tem o objetivo de apresentar uma metodologia para o planejamento de implementações de aprendizagem significativas em ambientes virtuais de aprendizagem utilizando Modelagem e Notação de Processo de Negócios (Business Process Model and Notation-BPMN).

Estruturalmente, o artigo está dividido em quatro seções. Após as considerações introdutórias, na seção seguinte é apresentada a fundamentação teórica. Na terceira seção é apresentada a metodologia de implementação da teoria de aprendizagem significativa no Moodle. Por fim, na seção quatro são apresentadas as considerações finais desse artigo.

\section{Fundamentação teórica}

A metodologia a ser abordada durante o estudo que configura a base para esse trabalho foi a teoria da aprendizagem significativa, tal como proposto por David Ausubel, onde se considera três tipos gerais de aprendizagem: a cognitiva, a afetiva e a psicomotora. A primeira é o resultado do armazenamento de informações organizadamente na estrutura cognitiva do sujeito. A segunda está ligada aos sentimentos e representações que o sujeito cria deles e a terceira envolve respostas musculares e depende do treino e da prática [Lara e Sousa 2009]. Esta visão retrata que o fator que mais influencia a aprendizagem significativa é o conjunto de conhecimentos prévios do aluno, a organização que ele já possui, a qual deverá servir como ponto de ancoragem para as novas informações; quanto mais claros, inclusivos e disponíveis forem tais conhecimentos prévios, tanto melhor será o nível de aprendizagem.

Em ambientes de ensino na modalidade a distância (EAD), a metodologia significativa é focada na criação de uma parceria entre o professor e o aluno com o 
objetivo de atingir o conhecimento. Nesse caso, o aluno assume o papel de ator principal e o professor assume o papel de mediador, e estimulador, do processo do ensino com foco na aprendizagem. A ideia é estimular a autonomia intelectual dos alunos por meio de atividades, que são previamente planejadas pelo professor, para promover o uso de habilidade de pensamento, como interpretar, desenvolver uma análise, fazer síntese de temas diversos e como classificar, relacionar e comparar [Rolim e Scaramuzza 2016].

Quanto mais perto dessa perspectiva, da aprendizagem significativa, mais os Ambientes Virtuais de Aprendizagem (AVAs) irão apresentar um melhor resultado relativo ao retorno esperado às reais necessidades do aluno, através das questões essenciais que permeiam os conteúdos a serem ministrados [Simão et al. 2017]. Estes conteúdos podem ser disponibilizados por diversas metodologias ou técnicas.

Uma abordagem de disponibilização de conteúdos para alunos após uma série de questionários niveladores terem sidos aplicados é apresentado em Lara e Souza [2009], sugerindo como uma metodologia válida para ajudar a entender o nivelamento de alunos antes da aplicação de uma metodologia significativa. Ao passo que, em seguida, pode ser adotada uma metodologia dialética como referencial pedagógico, que sustente a construção do conhecimento em três fases: Mobilização, Construção e Síntese, como apresentado por [Prado et al. 2012].

Quanto às diversas técnicas para a disponibilização de conteúdo e formas de interação entre os alunos que possibilitem a implantação da aprendizagem significativa em AVAs, pode-se citar: blogs, wikis, podcasts, e-portfolios, social networking, social bookmarking, photo sharing, second life, online forums, vídeo messaging, YouTube, audiographics, dentre outras, mescladas em atividades comunicacionais síncronas e assíncronas [Messa 2010; Rolim e Scaramuzza 2016]; além da possibilidade de elaboração de materiais didáticos com o uso de Design Educacional [Silva et al. 2018] ou mesmo uma produção específica de objetos de aprendizagem baseadas na aprendizagem significativa que possibilite a compreensão da dinâmica entre o uso da metodologia ativa e a produção de mídias Digitais Educacionais [Silva et al. 2019].

\section{Metodologia para implementação de Aprendizagem Significativa no Moodle}

Para a metodologia proposta neste artigo foi adotado o Modular Object-Oriented Dynamic Learning Environment (Moodle) como Ambiente Virtual de Aprendizagem, por ser um software de fonte aberta e livre, além de ser amplamente utilizado mundialmente [Melo et al. 2019]. Este ambiente de aprendizagem possui uma estrutura que permite a criação de sequências de "trilhas" a serem exploradas pelos os alunos, de acordo com o seus interesses e necessidades, que são percorridas em uma ordem prédefinida pelo professor, que irá estruturar o conteúdo na plataforma. Então, ao interagir com o Moodle, baseado nos seus conhecimentos prévios, o aluno pode estabelecer correlações entre os diversos assuntos inter-relacionados, dada uma exploração ativa que colabora na ampliação de seus conhecimentos sobre um determinado tema de estudo, na sua capacidade associativa de ideias e também para adquirir novos aprendizados [Messa 2010; Simão et al. 2017]. 
Desta forma, esta metodologia proposta é baseada na estrutura de Rede Conceitual existente no Moodle, como ilustra a Figura 1, onde é possível identificar todos os conceitos cognitivos que são desenvolvidos pelos alunos, destacando-se as características tecnológica e educacional não sequencial e não hierárquica dos percursos disponíveis para a construção do conhecimento, ajustáveis conforme as necessidades do professor.

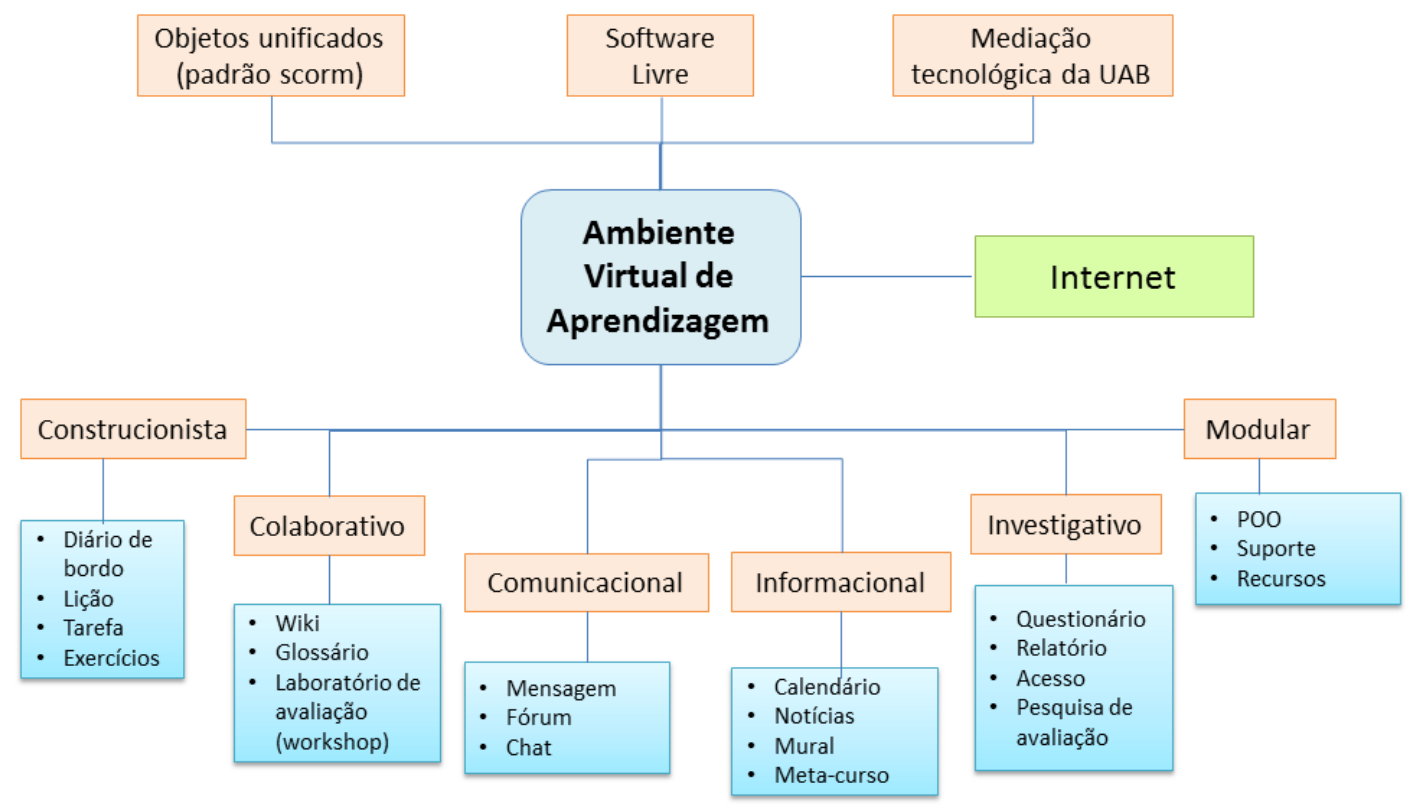

Figura 1: Ambiente virtual de aprendizagem

Dentro da especialidade construcionista será desenvolvido o recurso "lição" e suas competências. Ela funciona de maneira diferenciada quando comparada aos fóruns e wikis, entretanto permite uma boa flexibilidade de interação por parte dos professores. Além disso, a "lição" é o único recurso do Moodle que permite a configuração de prérequisitos e contempla a possibilidade da quebra estrutural linear das aulas, permitindo que o aluno percorra por textos e materiais em ordem personalizada. A Figura 2 ilustra de maneira objetiva e simples a estruturação de uma "lição" genérica no Moodle.

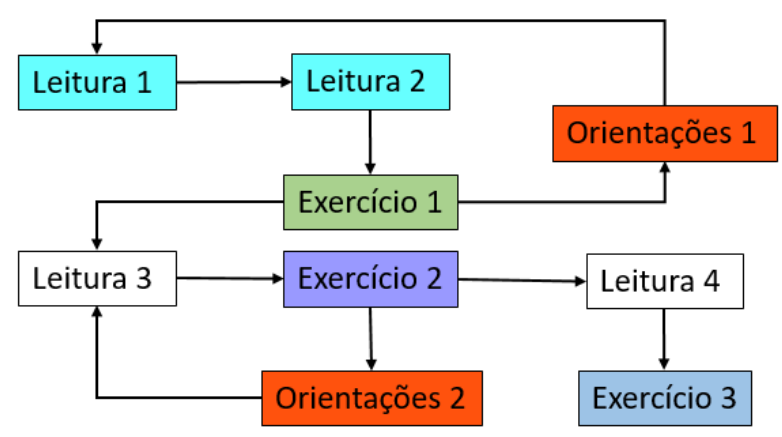

Figura 2: Estrutura de uma "lição" no Moodle 
Sua lógica de funcionamento ocorre a partir da especificação de um pequeno texto, seguido de uma pergunta de fixação sobre aquele conteúdo. Caso, o aluno acerte a pergunta, poderá avançar na lição e ler um novo texto ou assistir um vídeo. Caso a resposta esteja errada, é possível direcioná-lo para um texto corretivo, com a explicação detalhada do erro e sugerindo uma nova leitura.

O BPMN foi utilizado para o mapeamento dos processos relacionado à implementação desta metodologia. Desta forma, para a criação da estrutura de uma disciplina no desenvolver da aprendizagem significativa, a metodologia da implementação pode ser descrita em três fases distintas e englobam as seguintes etapas: experiência, reflexão, pensamento e Sintetização do conhecimento. Para representar essas etapas, foram estruturadas as fases 1, 2, 3, onde a Fase 1 engloba as etapas de experiência e reflexão, a Fase 2 representa a etapa de pensamento e, por fim, a Fase 3 que representa sintetização do conhecimento.

\subsection{Fase 1 - Experiência e Reflexão}

Nesta primeira fase inicia-se o processo de avaliação do conhecimento e experiência prévia do aluno, onde o professor é responsável por disponibilizar uma atividade de fixação (lição) que contemple os materiais de leitura a ser previamente acessados pelos alunos da disciplina e que despertem o interesse e mobilização para o estudo do mesmo.

Esta Fase tem como objetivo reter os conhecimentos repassados durante esse momento inicial através dos materiais de conteúdo disponibilizados pelo professor, como ilustra a Figura 3. Com esta atividade, é possível que o aluno possa testar seus próprios conhecimentos e, caso encontre algum tipo de dificuldade durante a execução da atividade, o professor atua como o principal facilitador para orientar nos questionamentos.

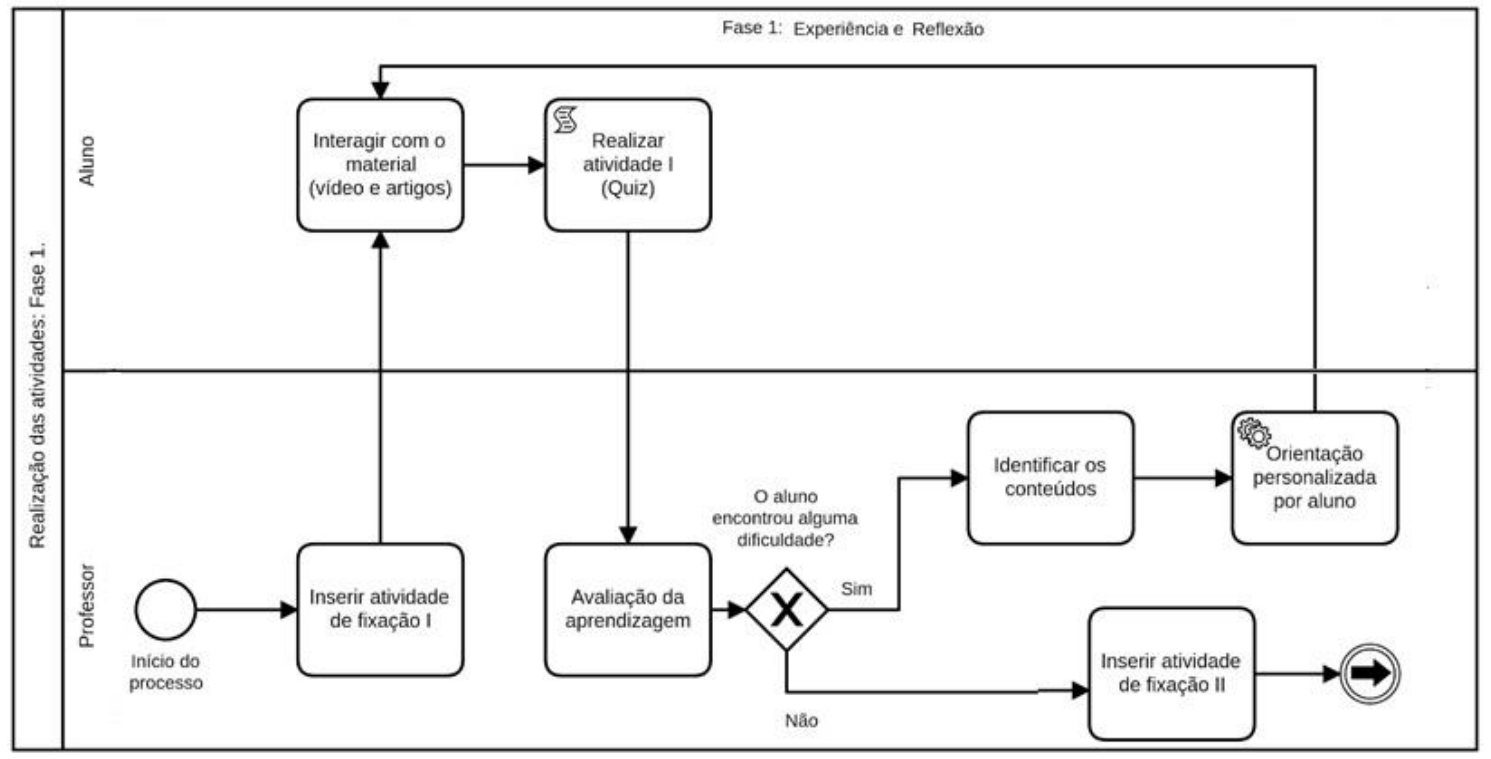

Figura 3: Modelo BPM da abordagem significativa, Fase 1, por meio de lições 


\subsection{Fase 2 - Pensamento}

A segunda fase compreende a construção do conhecimento. Nesta fase foi proposta uma nova atividade aplicada pelo professor, seguindo o mesmo esquema ilustrado na fase anterior (Fase 1), buscando fixar o conteúdo disponibilizado para leitura. Diferentemente da fase anterior, aqui é extremamente importante que o aluno tenha concluído, de maneira proveitosa, a fase anterior (Experiência e Reflexão) para que seja possível identificar o progresso do aluno com relação ao progresso do conhecimento, buscando entender a influência da metodologia e sua eficácia no processo, conforme demonstrado na Figura 4.

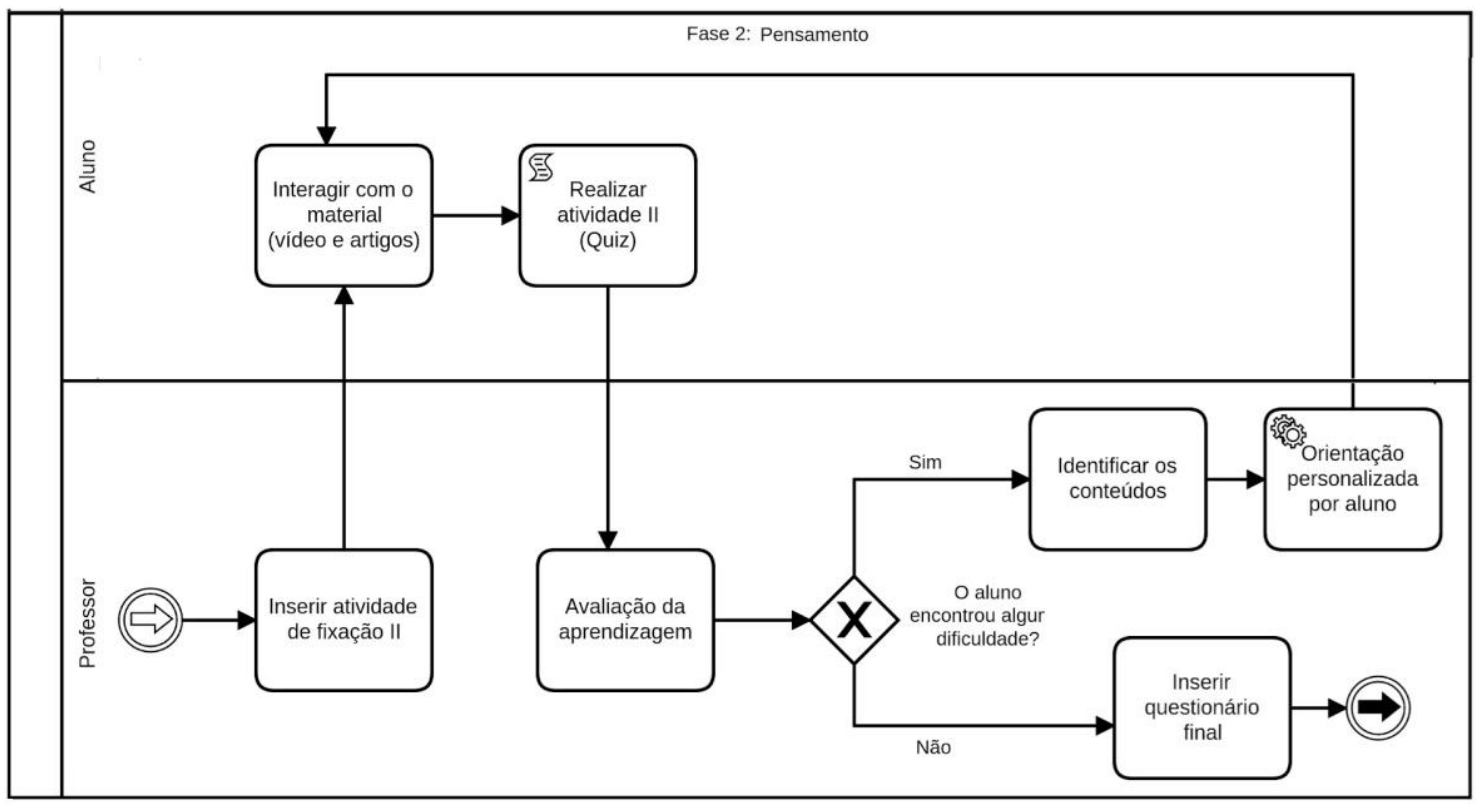

Figura 4: Modelo BPM da abordagem significativa, Fase 2, por meio de lições

Apesar desta modelagem conter apenas duas atividades (Fase 1 e Fase 2 respectivamente), fica a critério do professor desenvolver quantas atividades forem necessárias para passar todo o conhecimento a respeito de um determinado assunto, não necessariamente se limitando ao exemplo demonstrado neste artigo.

\subsection{Fase 3 - Sintetização do conhecimento}

Por fim, a terceira fase compreende a síntese do conhecimento, e conclui o ciclo de fixação do conteúdo disponibilizado pelo professor. Com a intenção de estimular o estudante a resgatar e sintetizar o que foi apreendido nas fases anteriores, utiliza-se um questionário final que busca avaliar todo o progresso de ponta a ponta do aluno com relação ao primeiro momento do ciclo. Esta fase possibilita o professor analisar os resultados gerados por cada um dos alunos a fim de investigar seus pontos fracos e fortes, entender os impactos da metodologia no processo de aprendizagem, assim também como produzir um feedback assistivo e significativo para todos os alunos de uma maneira geral, conforme ilustra a Figura 5. 


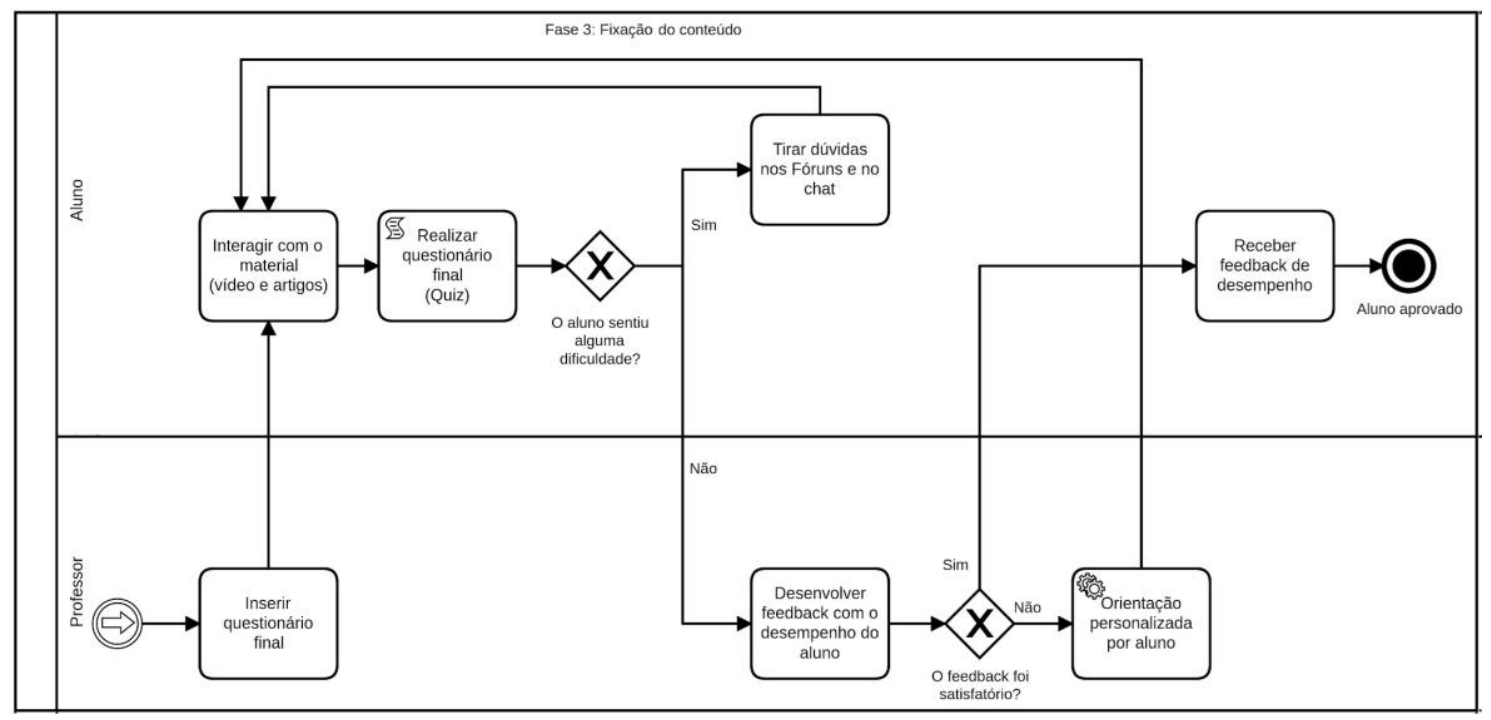

Figura 5: Modelo BPM da abordagem significativa, Fase 3, por meio de lições

\subsection{Estruturação das Atividades Significativas}

Uma vez compreendida a modelagem metodológica proposta neste artigo, faz-se necessário para sua implementação, a compreensão do processo de estruturação de atividades significativas. Este processo inicia-se com a configuração geral da lição, que é composta basicamente por um conjunto de atividades. Depois que a lição está configurada, pode-se adicionar atividades no conjunto. Cada atividade será criada respeitando uma ordem evolutiva das fases da aprendizagem significativa, garantindo assim uma melhor transmissão do conhecimento e respeitando das diretrizes da metodologia. É importante destacar que, além de disponibilizar materiais de apoio para os alunos, é fundamental que o professor elabore explicações e orientações do funcionamento de cada lição e/ou atividade, evitando dúvidas e dificuldades iniciais.

Sobre o conteúdo das atividades, cada uma delas pode apresentar os mesmos itens de uma página web, como material oriundo de sites sociais como o Youtube, por exemplo, sem mencionar o uso de apresentações condensado.

Cada uma das atividades apresenta uma opção para adicionar perguntas ao conteúdo, com várias opções de resposta. Para cada alternativa que o aluno tem de responder, o professor ou tutor pode adicionar um feedback personalizado e também direcionar a navegação para outras páginas. É importante salientar que o acompanhamento individualizado de cada aluno permite um melhor aproveitamento do aprendizado para a turma, visto que as pessoas têm sua individualidade e jeito próprio de absorver conteúdos e feedbacks. Assim deve-se utilizar técnicas de feedback, como a sanduíche [Leite et al. 2018], para minimizar o desestímulo do aluno ao receber a um feedback negativo.

A seguir deve-se disponibilizar o recurso para os alunos, sem esquecer-se de oferecer algum tipo de orientação para os mesmos sobre o funcionamento desse tipo de atividade. Se os alunos estiverem acostumados apenas com o uso de questionários, a estrutura de uma lição pode ser um pouco confusa para usuários sem o conhecimento no recurso e cabe ao professor orientá-lo quanto a utilização. 
Além da estrutura de lições, e dentro do caráter comunicacional, o professor pode trabalhar com o Chat e Fórum. O Chat é uma ferramenta muito conhecida nas comunidades de aprendizagem. No Moodle, o professor pode criar salas de bate papo, ativando a edição e escolhendo Chat na lista de atividades da seção à qual o bate papo irá pertencer. A realização dos chats com objetivos pedagógicos deve estabelecer algumas regras que serão construídas junto com o grupo, para possibilitar a participação de todos, bem como o atendimento das demandas dos usuários. Essas regras não têm o objetivo de inibir a participação dos alunos. Ao contrário, o objetivo é dar voz a todo o grupo, possibilitando a construção coletiva do conhecimento.

O Fórum tem por objetivo encorajar a discussão e promover um conhecimento coletivo, funcionando apenas se: a) os participantes sentirem que há uma necessidade/razão de participar e eles terão algum ganho com a experiência como incentivos para aprendizagem; b) o clima de comunidade e propósito pode ser fomentada entre participantes. Esta sensação de comunidade pode ser alimentada pela iniciativa e apoio do tutor/professor, ou primariamente por intermédio dos próprios estudantes/participantes dependendo da intenção da atividade. O Moodle possui quatro tipos de fóruns, cada um com ligeiras diferenças de apresentação e proposta. Dentre estes, o fórum de Perguntas e Respostas oculta as respostas de outros alunos até que se responda à pergunta. Após a postagem inicial, os alunos podem ver e comentar as respostas de outros alunos e, portanto, esse recurso garante igual oportunidade aos alunos e encoraja o raciocínio independente.

\section{Considerações Finais}

A aprendizagem significativa é uma teoria de ensino/aprendizagem que se tornou conhecida no final da década de 60 e que apresenta conceitos interessantes quando se trata do processo educacional de um indivíduo. Com o uso cada vez mais cotidiano das TDICs na modalidade da Educação a Distância, faz-se necessário que Ambientes Virtuais de Aprendizagem utilizem metodologias e teorias que possibilitem um aprendizado mais significativo e ativo para os alunos.

Devido a necessidade da compreensão do processo de implementação de uma sala de aula virtual considerando a aprendizagem significativa e independentemente de área de conhecimento de um professor, neste artigo foi apresentado uma metodologia para o planejamento de implementações de aprendizagem significativas em ambientes virtuais de aprendizagem utilizando Modelagem de Processo de Negócios.

Uma vez definida a metodologia, verificou-se que a teoria de aprendizagem significativa pode ser adotada de forma simples, sem a necessidade de desenvolvimento de artefatos educacionais específicos para AVAs e independentemente da área de estudo e aplicação.

Porém, no que tange ao professor, será necessário um planejamento prévio de todo o processo da construção de conteúdo e estratégias que possibilitem uma reavaliação do aprendizado do aluno de forma contínua ao longo do tempo. Desta forma, haverá um custo maior no tempo de preparo das atividades e principalmente no planejamento, e execução de um feedback personalizado. 
Por fim, a adoção para prática de aprendizagem significativa em AVAs pode ser iniciada individualmente, por ações isoladas de professores, ou com um maior impacto, quando estimulada pelas instituições, orientando e capacitando seus professores na construção de suas disciplinas apoiadas na teoria significativa e implementadas em um AVA de forma simples, resultando assim, em uma educação mais construtiva e duradoura para a sociedade.

\section{Referências}

Carvalho, M.M., Vasconcelis, J.G e Marçal, E.(2018) "Impactos da Educação a Distância na Vida dos Egressos: Uma Análise Comparativa de Estudos na Universidade Averta do Brasil". In: III Congresso sobre Tecnologias na Educação (Ctrl+E 2018). Fortaleza.

Lara, A. Elisa; Sousa, C. M. S. G.(2009) “O Processo De Construção E De Uso De Um Material Potencialmente Significativo Visando A Aprendizagem Significativa Em Tópicos De Colisões: Apresentações De Slides E Um Ambiente Virtual De Aprendizagem. Experiências em Ensino de Ciências , [s. 1.], v. 4, ed. 2, p. pp.61-82.

Leite, A.S., Nascimento, V.C. e Matteu, D.(2018) “O Feedback nas Organizações: Técnicas e estratégias para fornecer um feedback". Revista de Humanidades, Tecnologia e Cultura. Vol 8, n.1.

Melo, S.B., Junior, A.S., Rodrigues, E.C.C. e Lima, C.M.(2019) "Avaliação de um Ambiente Virtual de Aprendizagem da Plataforma Moodle". IX Congresso Brasileiro de Engenharia de Produção. Ponte Grossa-PR.

Messa, Wilmara Cruz. (2010) Utilização de Ambientes Virtuais de Aprendizagem AVAS: A Busca por uma Aprendizagem Significativa. Revista Brasileira de Aprendizagem Aberta e a Distância, vol.9.

Prado, C., Vaz, D. R. e Almeida, D. M.(2012) “Teoria da Aprendizagem Significativa: elaboração e avaliação de aula virtual na plataforma Moodle”. Revista Brasileira de Enfermagem, vol. 64, núm. 6, pp. 1114-1121. Associação Brasileira de Enfermagem.

Rolim, A. T. e Scaramuzza, B. C.(2016) Aprendizagem significativa em ambientes virtuais de aprendizagem. - Revista Do Programa De Pós-Graduação Em Educação Mestrado - Univ. do Sul de Santa Catarina. Unisul, Tubarão, v.10, n. Especial, p. $182-195$.

Scaramuzza, Bruno Cézar.(2015) "Universidade Corporativa: Educação Continuada e Mediação Tecnológica”. Dissertação de Mestrado UNOPAR, Londrina-PR.Silva, E.C., Reis, J.T.C. e Martins, R.S.(2018) "Design Educacional na elaboração de materiais didáticos para cursos online: uma proposta de formação docente". III Congresso sobre Tecnologias na Educação (Ctrl+E), Fortaleza.

Silva, E.C., Reis, J.T.C. e Martins, R.S.(2018) "Design Educacional na Elaboração de materiais didáticos para cursos online: uma proposta de Formação Docente". In:III Congresso sobre Tecnologias na Educação (Ctrl+E), Fortaleza.

Silva, D.C.M.S., Sobrinho, I., Dutra, K.L., Lima, T. e Silva, W.B.(2019) "As Metodologias Ativas e a Aprendizagem Significativa com determinantes na produção 
de Tecnologias Educacionais". IV Congresso sobre Tecnologias na Educação $(\mathrm{Ctrl}+\mathrm{E})$, Recife.

Simão, José Pedro Schardosim et al.(2017)“Inovação Educativa e Usabilidade em Ambientes Virtuais de Aprendizagem”. Anais do Simpósio Ibero-Americano de Tecnologias Educacionais, [S.1.], p. 45-52, Araranguá. 